\title{
Sorting classes
}

\author{
M. H. Albert \\ Department of Computer Science \\ University of Otago \\ malbert@cs . otago.ac.nz \\ M. D. Atkinson \\ Department of Computer Science \\ University of Otago \\ mike@cs.otago.ac.nz \\ D. A. Holton \\ Department of Mathematics and Statistics \\ University of Otago \\ dholton@math.otago.ac.nz
}

\author{
R. E. L. Aldred \\ Department of Mathematics and Statistics \\ University of Otago \\ raldred@math.otago.ac.nz \\ C. C. Handley \\ Department of Computer Science \\ University of Otago \\ chandley@cs.otago.ac.nz \\ D. J. McCaughan \\ Department of Mathematics and Statistics \\ University of Otago \\ dmccaughan@math.otago.ac.nz
}

\author{
H. van Ditmarsch \\ Department of Computer Science \\ University of Otago \\ hans@cs.otago.ac.nz
}

Submitted: Dec 20, 2004; Accepted: Jun 17, 2005; Published: Jun 26, 2005

Mathematics Subject Classifications: 05A15, 05A16

\begin{abstract}
Weak and strong sorting classes are pattern-closed classes that are also closed downwards under the weak and strong orders on permutations. They are studied using partial orders that capture both the subpermutation order and the weak or strong order. In both cases they can be characterised by forbidden permutations in the appropriate order. The connection with the corresponding forbidden permutations in pattern-closed classes is explored. Enumerative results are found in both cases.
\end{abstract}

\section{Introduction}

A permutation $\pi$ is said to be a subpermutation of a permutation $\sigma$ (or to be involved in $\sigma)$ if $\sigma$ has a subsequence that is ordered in the same relative way as $\pi$. For example 231 is a subpermutation of 35412 because of its subsequence 351 which has the same pattern 
as 231. We say that $\sigma$ avoids $\pi$ if $\pi$ is not a subpermutation of $\sigma$. The developing theory of permutation patterns is now a well-established part of combinatorics (see, for example, $[12])$.

This theory was originally motivated by the study of the sortable permutations associated with various computing devices (abstract data types such as stacks and deques [8], token passing networks [3], or hardware switches [2]). All these devices have the property that, if they are able to sort a sequence $\sigma$, then they are able to sort any subsequence of $\sigma$.

This subsequence property (that subsequences of sortable sequences are themselves sortable) is a very natural one to postulate of a sorting device. It is exactly this property that guarantees that the set of sortable permutations is closed under taking subpermutations. But there are other natural properties that a sorting device might have. We are particularly interested in the following two. Both of them reflect the idea that "more sorted" versions of sortable sequences should themselves be sortable.

1. If $s_{1} s_{2} \ldots s_{n}$ is sortable and $s_{i}>s_{i+1}$ then $s_{1} s_{2} \ldots s_{i-1} s_{i+1} s_{i} \ldots s_{n}$ is sortable, and

2. If $s_{1} s_{2} \ldots s_{n}$ is sortable and $s_{i}>s_{j}$ where $i<j$ then

$$
s_{1} s_{2} \ldots s_{i-1} s_{j} s_{i+1} \ldots s_{j-1} s_{i} s_{j+1} \ldots s_{n}
$$

is sortable.

For the moment we call these the weak and strong exchange properties (the second obviously implies the first). The weak exchange property would hold for sorting devices that operated by exchanging adjacent out of order pairs while the strong exchange property would hold if arbitrary out of order pairs could be exchanged. Our paper is about the interaction between each of these properties and the subsequence property.

We shall study this interaction using various (partial) orders on the set $\Omega$ of all (finite) permutations. Since we shall be considering several partial orders on $\Omega$ we shall write $\sigma \mathcal{P} \tau$ when we mean that $\sigma \leq \tau$ in the partial order $\mathcal{P}$; this avoids the confusion of the symbol " $\leq$ " being adorned by various subscripts. In the same spirit we write $\sigma \overline{\mathcal{P}} \tau$ to mean $\sigma \not \leq \tau$ in $\mathcal{P}$.

All the partial orders we study will satisfy the minimum condition (that is, all properly descending chains are finite) and we shall assume this from now on.

If $\mathcal{P}$ is a partial order on $\Omega$ the lower ideals of $\mathcal{P}$ are those subsets $X$ of $\Omega$ with the property

$$
\beta \in X \text { and } \alpha \mathcal{P} \beta \Longrightarrow \alpha \in X \text {. }
$$

Since $\mathcal{P}$ satisfies the minimum condition such a lower ideal can be studied through the set $b(X)$ of minimal permutations of $\Omega \backslash X$. Obviously $b(X)$ determines $X$ uniquely since

$$
X=\{\beta \mid \alpha \overline{\mathcal{P}} \beta \text { for all } \alpha \in b(X)\} .
$$

In the classical study of permutation patterns we use the subpermutation order that we denote by $\mathcal{I}$ (standing for involvement). The lower ideals of $\mathcal{I}$ are generally the central 
objects of study and are called closed classes. If $X$ is a closed class then $b(X)$ is called the basis of $X$. Indeed the most common way of describing a closed class is by giving its basis (and therefore defining it by avoided patterns). We write av $(B)$ to denote the set of permutations which avoid all the permutations of the set $B$. If a closed class is not given in this way then, often, the first question is to determine the basis. A second question, perhaps of even greater interest, is to enumerate the class; in other words, to determine by formula, recurrence or generating function how many permutations it has of each length.

However, these questions can be posed for any partial order on $\Omega$ and much of our paper is devoted to answering them for orders that capture the subsequence property and the weak or strong exchange properties.

A closed class is called a weak sorting class if it has the weak exchange property and a strong sorting class if it has the strong exchange property.

Our aim is to set up a framework within which these two notions can be investigated and to exploit this framework by proving some initial results about them. We shall begin by investigating the two natural analogues of the subpermutation order that are appropriate for these two concepts. In particular there are natural notions of a basis for each type of sorting class; we shall explore how the basis of a sorting class is related to the ordinary basis and use this to derive enumerative results. In the remainder of this section we set up the machinery for studying sorting classes and then survey the main results of Sections 2 and 3 on weak and strong sorting classes respectively.

The terms 'weak' and 'strong' have been chosen to recall two important orders on the set of permutations of length $n$ : the weak and strong orders. For completeness we shall give their definitions below (extended to the set $\Omega$ of permutations of all lengths). In these definitions and elsewhere in the paper we use Roman lower case letters for the individual symbols within a permutation and Greek lower case letters for sequences of zero or more symbols.

The weak order $\mathcal{W}$ on $\Omega$ can be defined as the transitive closure of the set of pairs

$$
\mathcal{W}_{0}=\{(\lambda r s \mu, \lambda s r \mu) \mid r<s\}
$$

The strong order $\mathcal{S}$ on $\Omega$ can be defined as the transitive closure of the set of pairs

$$
\mathcal{S}_{0}=\{(\lambda r \mu s \nu, \lambda s \mu r \nu) \mid r<s\} .
$$

Notice that, for both $\mathcal{W}$ and $\mathcal{S}$, only permutations of equal length can be comparable.

The subpermutation order $\mathcal{I}$ on $\Omega$ can be defined as the transitive closure of the set of pairs

$$
\mathcal{I}_{0}=\left\{\left(\lambda \mu, \lambda^{\prime} r \mu^{\prime}\right)\right\}
$$

where $\lambda^{\prime} \mu^{\prime}$ is order isomorphic to $\lambda \mu$.

Weak (respectively, strong) sorting classes are the lower ideals in the partial order defined by the transitive closure of $\mathcal{I} \cup \mathcal{W}$ (respectively $\mathcal{I} \cup \mathcal{S}$ ) and so can be studied using the same machinery that has been used for arbitrary closed classes, adapted to the appropriate order. 
We begin by giving a simple description of these transitive closures. In this description we denote the relational composition of two partial orders by juxtaposition.

Lemma 1 The transitive closure of $\mathcal{I} \cup \mathcal{W}$ is $\mathcal{I} \mathcal{W}$ while that of $\mathcal{I} \cup \mathcal{S}$ is $\mathcal{I} \mathcal{S}$. In fact $\mathcal{W I}=\mathcal{I} \mathcal{W}$ while $\mathcal{S I}$ is strictly included in $\mathcal{I S}$.

Proof: Suppose that $\alpha \mathcal{I} \beta \mathcal{W}_{0} \gamma$ represents a pair $(\alpha, \gamma)$ of the relation $\mathcal{I W}_{0}$. Let $\alpha=a_{1} a_{2} \ldots$ and let $a_{1}^{\prime} a_{2}^{\prime} \ldots$ be a subsequence of $\beta$ order isomorphic to $\alpha$. Let $x y$ be the two adjacent symbols of $\beta$ that become $y x$ in $\gamma$. If none or one of these is one of the $a_{i}^{\prime}$ then $\alpha \mathcal{I} \gamma$. If both of them are among $a_{1}^{\prime} a_{2}^{\prime} \ldots$ then they must be $a_{i}^{\prime}$ and $a_{i+1}^{\prime}$ for some $i$. Let $\beta^{\prime}$ be the result of exchanging $a_{i}$ and $a_{i+1}$ in $\alpha$; then we have $\alpha \mathcal{W} \beta^{\prime} \mathcal{I} \gamma$. This proves that $\mathcal{I} \mathcal{W}_{0} \subseteq \mathcal{W} \mathcal{I}$ and it follows readily that $\mathcal{I} \mathcal{W}_{0}^{t} \subseteq \mathcal{W} \mathcal{I}$ for all $t$ and hence that $\mathcal{I} \mathcal{W} \subseteq \mathcal{W I}$.

To prove the opposite inclusion suppose that $\alpha \mathcal{W}_{0} \beta \mathcal{I}_{0} \gamma$ represents a pair $(\alpha, \gamma)$ of the relation $\mathcal{W}_{0} \mathcal{I}_{0}$. Then we have

$$
\begin{aligned}
\alpha & =\theta a b \phi \\
\beta & =\theta b a \phi
\end{aligned}
$$

and $\gamma$ is obtained from $\beta$ by inserting an extra symbol $x$ (with appropriate renumbering of the symbols larger than $x$ ).

If $x$ does not occur between $b$ and $a$ then we can consider $\gamma$ to be obtained from $\alpha$ by first inserting $x$ and then swapping $a$ and $b$; so, in this case, $\alpha \mathcal{I}_{0} \mathcal{W} \gamma$. If $x$ occurs between $b$ and $a$ then, depending on the value of $x$, we define $\xi$ as either $\theta x a b \phi, \theta a x b \phi, \theta a b x \phi$ so that the three symbols $a, b, x$ come in increasing order. Then

\section{$\theta a b \phi \mathcal{I}_{0} \xi \mathcal{W} \theta b x a \phi$}

and so, again, $\alpha \mathcal{I}_{0} \mathcal{W} \gamma$

We have proved that $\mathcal{W}_{0} \mathcal{I}_{0} \subseteq \mathcal{I}_{0} \mathcal{W}$ and it readily follows that $\mathcal{W} \mathcal{I}_{0} \subseteq \mathcal{I}_{0} \mathcal{W}$, and then that $\mathcal{W} \mathcal{I} \subseteq \mathcal{I} \mathcal{W}$. The transitive closure of $\mathcal{I} \cup \mathcal{W}$ is, by definition,

$$
\bigcup_{i=0}^{\infty}(\mathcal{I} \cup \mathcal{W})^{i} .
$$

However, $\mathcal{I}$ and $\mathcal{W}$ are transitively closed and $\mathcal{W} \mathcal{I} \subseteq \mathcal{I} \mathcal{W}$, and so this expression simplifies to $\mathcal{I} \mathcal{W}$.

Suppose now that $\alpha \mathcal{S}_{0} \beta \mathcal{I} \gamma$ represents a pair $(\alpha, \gamma)$ of the relation $\mathcal{S}_{0} \mathcal{I}$. Put $\alpha=$ $\lambda r \mu s \nu$ with $r<s$ and $\beta=\lambda s \mu r \nu$. Let $\lambda^{\prime} s^{\prime} \mu^{\prime} r^{\prime} \nu^{\prime}$ denote a subsequence of $\gamma$ order isomorphic to $\beta$. Consider the permutation $\gamma^{\prime}$ obtained from $\gamma$ by interchanging $s^{\prime}$ and $r^{\prime}$. Clearly $\alpha \mathcal{I} \gamma^{\prime} \mathcal{S} \gamma$. This shows that $\mathcal{S}_{0} \mathcal{I} \subseteq \mathcal{I} \mathcal{S}$. But then it follows, as above, that $\mathcal{S I} \subseteq \mathcal{I} \mathcal{S}$. However $321 \mathcal{I} 1432 \mathcal{S} 3412$ yet there exists no permutation $\theta$ with $321 \mathcal{S} \theta \mathcal{I} 3412$; therefore the inclusion is strict.

It follows as above that $\mathcal{I S}$ is the transitive closure of $\mathcal{I} \cup \mathcal{S}$. 
The orders $\mathcal{I} \mathcal{W}$ and $\mathcal{I S}$ have fewer symmetries (2 and 4 respectively) than the subpermutation order (which has 8 ). In the following elementary result, if $\zeta=z_{1}, \ldots, z_{n}, \zeta^{*}$ denotes the 'reverse complement' of $\zeta$

$$
\zeta^{*}=n+1-z_{n}, n+1-z_{n-1, \ldots, n+1-z_{1} .}
$$

Lemma 2 Let $\xi, \zeta$ be permutations. Then

1. $\xi \mathcal{I W} \zeta \Longleftrightarrow \xi^{*} \mathcal{I} \mathcal{W} \zeta^{*}$, and

2. $\xi \mathcal{I S} \zeta \Longleftrightarrow \xi^{-1} \mathcal{I S} \zeta^{-1} \Longleftrightarrow \xi^{*} \mathcal{I S} \zeta^{*}$.

We have already noted that every closed class $X$ can be described by a forbidden pattern set $T$ as

$$
\operatorname{av}(T)=\{\sigma \mid \beta \overline{\mathcal{I}} \sigma \text { for all } \beta \in T\}
$$

We can describe weak and strong sorting classes in a similar way using the orders $\mathcal{I} \mathcal{W}$ and $\mathcal{I S}$. In other words, given a set $T$ of permutations we define

$$
\begin{aligned}
\operatorname{av}(T, \mathcal{I} \mathcal{W}) & =\{\sigma \mid \beta \overline{\mathcal{I} \mathcal{W}} \sigma \text { for all } \beta \in T\} . \\
\operatorname{av}(T, \mathcal{I} \mathcal{S}) & =\{\sigma \mid \beta \overline{\mathcal{I S}} \sigma \text { for all } \beta \in T\} .
\end{aligned}
$$

which are weak and strong sorting classes respectively. Every weak and strong sorting class $X$ can be defined in this way taking for $T$ that set of permutations minimal with respect to $\mathcal{I W}$ or $\mathcal{I S}$ not belonging to $X$. If $T$ is the minimal avoided set then it is tempting to call it the basis of the class it defines. Unfortunately that leads to a terminological ambiguity since both $\operatorname{av}(T, \mathcal{I} \mathcal{W})$ and $a v(T, \mathcal{I S})$ are pattern closed classes and so have bases in the ordinary sense. To avoid such confusion we shall use the terms weak basis and strong basis. However, two significant questions now arise. If we have defined a weak sorting class by its weak basis, what is its basis in the ordinary sense? Similarly for strong sorting classes, what is the connection between the strong basis and the ordinary basis?

In the next section, on weak sorting classes, we shall see that the first of these questions has a relatively simple answer. In that section we also give a general result about the weak sorting class defined by a basis that is the direct sum of two sets. We go on to enumerate weak sorting classes whose weak basis is a single permutation of length at most 4 .

In the final section, on strong sorting classes, we shall see that the ordinary basis is not easily found from the strong basis. Nevertheless we can define a process that constructs the ordinary basis from the strong basis; and we prove that the ordinary basis is finite if the strong basis is finite. We have used this process as a first step in enumerating strong sorting classes defined by a single strong basis element of length at most 4 . We shall give a summary of these results and some remarks on their proofs.

We also introduce a 2-parameter family of strong sorting classes denoted by $\mathcal{B}(r, s)$. These classes are important because every (proper) strong sorting class is contained in one (indeed infinitely many) of them. We shall show how the $\mathcal{B}(r, s)$ can be enumerated and give a structure theorem that expresses $\mathcal{B}(r, s)$ as a composition of very simple strong sorting classes. 


\section{Weak sorting classes}

Proposition 3 Let $T$ be a set of permutations and let

$$
T^{\prime}=\{\sigma \mid \tau \mathcal{W} \sigma \text { for some } \tau \in T\}
$$

(the upper weal closure of $T$ ). Then

$$
\operatorname{av}(T, \mathcal{I} \mathcal{W})=\operatorname{av}(T, \mathcal{W} \mathcal{I})=\operatorname{av}\left(T^{\prime}\right)
$$

Proof: The first equality is immediate from Lemma 1. To prove the second, first suppose that $\sigma \notin \operatorname{av}(T, \mathcal{W} \mathcal{I})$. Then, for some $\tau \in T$, we have $\tau \mathcal{W} \mathcal{I} \sigma$. Hence there exists $\tau^{\prime} \in T^{\prime}$ with $\tau \mathcal{W} \tau^{\prime} \mathcal{I} \sigma$. The final relation says that $\sigma \notin a v\left(T^{\prime}\right)$.

Conversely, suppose that $\sigma \notin a v\left(T^{\prime}\right)$. Then, for some $\tau^{\prime} \in T^{\prime}$, we have $\tau^{\prime} \mathcal{I} \sigma$. By definition of $T^{\prime}$ there exists $\tau \in T$ with $\tau \mathcal{W} \tau^{\prime}$. But then $\tau \mathcal{W} \mathcal{I} \sigma$ which means that $\sigma \notin \operatorname{av}(T, \mathcal{W I})$.

Corollary 4 The class av $(T)$ is a weak sorting class if and only if every permutation in the upward weak closure of $T$ involves a permutation of $T$.

Proof: Let $T^{\prime}$ be the upward weak closure of $T$. Then, by the previous proposition, $\operatorname{av}(T, \mathcal{I} \mathcal{W})=\operatorname{av}\left(T^{\prime}\right)$ and so $a v(T)$ is a weak sorting class if and only if $\operatorname{av}(T)=\operatorname{av}\left(T^{\prime}\right)$. The Corollary now follows.

Corollary 5 If a weak sorting class has a finite weak basis then its ordinary basis is also finite.

Proof: Let $T$ be the weak basis of a weak sorting class and let $T^{\prime}$ be its upward weak closure. Obviously, $T^{\prime}$ is finite if $T$ is finite. While $T^{\prime}$ may not be the ordinary basis of av $\left(T^{\prime}\right)$ (since it might not be an antichain) this ordinary basis just consists of the minimal elements of $T^{\prime}$ and so is finite.

To state the next result we need to recall the notion of the direct sum of two sets of permutations and some related terms. If $\alpha$ and $\beta$ are permutations of lengths $m$ and $n$ then $\alpha \oplus \beta$ is the permutation of length $m+n$ whose first $m$ symbols are all smaller than the last $n$ symbols, the first $m$ symbols comprise a sequence isomorphic to $\alpha$, and the last $n$ symbols comprise a sequence isomorphic to $\beta$. We extend this notion to sets $X$ and $Y$ of permutations by defining

$$
X \oplus Y=\{\alpha \oplus \beta \mid \alpha \in X, \beta \in Y\} .
$$

We also recall that a permutation is said to be indecomposable if it cannot be expressed as $\alpha \oplus \beta$. Every permutation has a unique expression in the form $\alpha_{1} \oplus \cdots \oplus \alpha_{k}$ where each $\alpha_{i}$ is indecomposable, and the $\alpha_{i}$ are called the components of $\alpha$. Closed classes whose basis elements are all indecomposable are somewhat easier to handle than arbitrary ones. This is because they have the property of being closed under direct sums and can be enumerated if their indecomposables can be enumerated [4]. 
Theorem 6 Let $R, S$ be the weak bases of weak sorting classes $\mathcal{A}, \mathcal{B}$ and let $\mathcal{C}$ be the weak sorting class whose weak basis is $T=R \oplus S$. Let $\left(a_{n}\right),\left(b_{n}\right),\left(c_{n}\right)$ be the enumeration sequences for $\mathcal{A}, \mathcal{B}, \mathcal{C}$ and let $a(t), b(t), c(t)$ be the associated exponential generating functions. Then

$$
c(t)=(t-1) a(t) b(t)+a(t)+b(t) .
$$

Proof: Let $R^{\prime}, S^{\prime}, T^{\prime}$ be the upward weak closures of $R, S, T$. By Proposition 3, we have $\mathcal{A}=\operatorname{av}\left(R^{\prime}\right), \mathcal{B}=\operatorname{av}\left(S^{\prime}\right)$, and $\mathcal{C}=\operatorname{av}\left(T^{\prime}\right)$. We can compute the structure of the permutations of $T^{\prime}$ using the property that they are in the upward weak closure of some $\rho \oplus \sigma(\rho \in R, \sigma \in S)$. Such permutations must be the union of two sequences $\rho^{\prime}, \sigma^{\prime}$ where

1. $\rho^{\prime}<\sigma^{\prime}$, and

2. $\rho^{\prime}, \sigma^{\prime}$ are (order isomorphic to) permutations of $R^{\prime}, S^{\prime}$.

Conversely, every such permutation is in the upward weak closure of some $\rho \oplus \sigma \in R \oplus S$ and so lies in $T^{\prime}$.

From this description we can determine the structure of permutations in $\mathcal{C}$. We describe them using a temporary notation: if $\pi$ is a permutation then $\pi \|_{[i \cdots j]}$ denotes the subsequence of $\pi$ whose values comprise the interval $[i \cdots j]$. All permutations in $\mathcal{C}$ of length $n$ will belong to one of the following two types:

- permutations belonging to $\mathcal{A}$;

- permutations $\pi$ not belonging to $\mathcal{A}$ which have the property that if $k$ is the minimum value such that $\pi \|_{[1 \cdots k]} \notin \mathcal{A}$ then $\pi \|_{[(k+1) \cdots n]} \in \mathcal{B}$.

Consider the collection of permutations not belonging to $\mathcal{A}$ but which have the property that the permutation resulting from the deletion of their maximum symbol does lie in $\mathcal{A}$. If we define $\hat{a}_{n}$ to be the number of permutations of this type of length $n$ then it is easy to see that:

$$
\hat{a}_{n}=n a_{n-1}-a_{n}
$$

since the first term on the right hand side counts the number of ways of adding a new maximum to a permutation in $\mathcal{A}$ of length $n-1$ while the second term subtracts the number of ways to do this which still result in a permutation in $\mathcal{A}$.

The description of the permutations in $\mathcal{C}$ then shows that:

$$
c_{n}=a_{n}+\sum_{k=0}^{n}\left(\begin{array}{l}
n \\
k
\end{array}\right) \hat{a}_{k} b_{n-k}
$$

and the theorem follows by comparison of series.

So far as we know this is the first appearance of exponential generating functions in pattern class enumeration. Notice from the form of the result that av $(R \oplus S, \mathcal{I} \mathcal{W})$ and av $(S \oplus R, \mathcal{I} \mathcal{W})$ are equinumerous. 
Proposition 3 shows that we can enumerate weak sorting classes using the various techniques that have been developed for ordinary closed classes. We shall begin these enumerative studies by looking at classes with a single basis permutation of length 3 or 4. The length 3 case is virtually trivial. By Lemma 2 we may restrict our attention to the permutations $123,132,231,321$ and we have

Proposition 7 The classes av $(123, \mathcal{I} \mathcal{W})$, av $(132, \mathcal{I} \mathcal{W})$, av $(231, \mathcal{I} \mathcal{W})$, av $(321, \mathcal{I} \mathcal{W})$ are enumerated by, respectively

1. $a_{n}=0$ for all $n \geq 3$,

2. $n$,

3. $2^{n-1}$,

4. the Catalan numbers.

For length 4 there is considerably more to do but Theorem 6 handles many of the cases. To within symmetry we have 16 permutations which, for discussion purposes, we have grouped into 4 families:

(i) 1234 ;

(ii) $2134,1324,2314,3124,3214,2143$;

(iii) 4231, 3421, 4321;

(iv) $2341,2413,3142,2431,3241,3412$.

The single permutation of the first family defines a finite class. The permutations of the second family are all handled by applying Theorem 6 and this gives the following enumerative formulae (valid for all $n \geq 2$ ):

\begin{tabular}{llllll}
2134 & 1324 & 2314 & 3124 & 3214 & 2143 \\
\hline$n(n-1)$ & $n(n-1)$ & $n 2^{n-2}$ & $n 2^{n-2}$ & $\left(\begin{array}{c}2 n-2 \\
n-1\end{array}\right)$ & $n 2^{n-1}-2^{n}+2$
\end{tabular}

The third family requires that we solve the enumeration problem for the closed classes with bases $\{4231,4321\},\{3421,4321\},\{4321\}$. The first of these (sequence A053617 of [11]) has an enumeration scheme in the sense of [14], the second gives the large Schröder numbers [9] and the third has been computed in [7].

The permutations in the last family present a series of different challenges. The easiest are 2341 and 3412. In these cases the classes are (in the notation of the next section) $\mathcal{B}(3,1)$ and $\mathcal{B}(2,2)$, and Proposition 20 gives us the recurrence relations $a_{n}=3 a_{n-1}$ and $a_{n}=4 a_{n-1}-2 a_{n-2}$ respectively. We treat the others in a series of lemmas.

Lemma 8 The class av $(2413, \mathcal{I} \mathcal{W})$ is enumerated by $\frac{1}{4}\left(3^{n}-2 n+3\right)$. 
Proof: The upward weak closure of 2413 is the set $\{2413,4213,2431,4231,4321\}$ but it is convenient instead to enumerate the class whose ordinary basis is $\{3142,3241,4132$, $4231,4321\}$ (the inverse class, which is not a weak sorting class). These basis elements tell us that if we have two disjoint descents then the latter lies entirely above the former; they also tell us that we can have at most two immediately adjacent descents.

Now it follows that two disjoint descents must lie in different components and so the indecomposables of the class begin with an increasing sequence, then have at most two down steps and end with an increasing sequence. The number of such having length $n$ is $n 2^{n-3}$ if $n \geq 3$. The ordinary generating function of the indecomposables is therefore

$$
g(t)=1+t+t^{2}+\sum_{n=3}^{\infty} n 2^{n-3} t^{n}
$$

and the full generating function is $\frac{1}{1-g(t)}$ from which the result follows.

Lemma 9 The class av $(3142, \mathcal{I} \mathcal{W})$ is enumerated by $\frac{1}{4}\left(3^{n}-2 n+3\right)$.

Proof: Let $b_{n}$ be the number of indecomposable permutations of length $n$ avoiding the 5 permutations $3142,3412,3421,4312,4321$ of the upward weak closure of 3142 . We shall show that $b_{n}=2 b_{n-1}+2^{n-3}$ from which follows $b_{n}=n 2^{n-3}$. Then the proof can be completed as in the previous lemma.

First note that, to avoid the permutations $3412,3421,4312,4321$, implies that symbol 1 or symbol 2 must occur in the first two positions. Therefore we can divide the indecomposable permutations into subsets (disjoint if $n>2$ ) as follows:

1. $F_{1}=\{\pi \mid \pi=1 \ldots\}$,

2. $F_{2}=\{\pi \mid \pi=2 \ldots\}$,

3. $S_{1}=\{\pi \mid \pi=t 1 \ldots\}$,

4. $S_{2}=\{\pi \mid \pi=t 2 \ldots\}$.

If $n>1$ then, by the indecomposability, $F_{1}$ is empty. Furthermore, if the initial symbol 2 is removed from a permutation of $F_{2}$ then the result remains indecomposable. Moreover, any indecomposable permutation of the class can be prefaced by a symbol 2 (incrementing the symbols larger than 2) and the result is not only in the class but is indecomposable. This shows that $\left|F_{2}\right|=b_{n-1}$. A similar argument proves that $\left|S_{2}\right|=b_{n-1}$.

Consider now a permutation $t 1 \ldots \in S_{1}$. Notice that $t \neq 2$ by indecomposability. We shall prove that $t=n$. If not, let $s$ be the rightmost symbol smaller than $t$ and write the permutation as $t 1 \alpha s \beta$. The avoidance of 3142 shows that $\alpha$ has no symbols larger than $t$, and $\beta$, by definition, has no symbols smaller than $t$. So $\beta$ consists precisely of the set $\{t+1, \ldots, n\}$ in some order, contradicting indecomposability as $t<n$. 

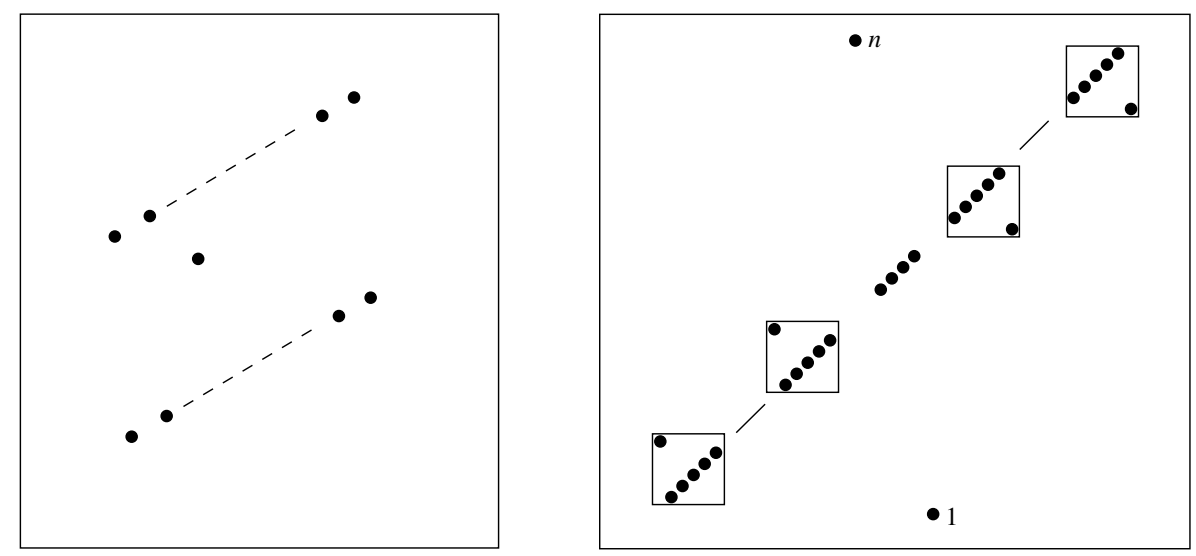

Figure 1: Indecomposable permutations in $\operatorname{av}(2413, \mathcal{I} \mathcal{W})$ and $a v(3142, \mathcal{I} \mathcal{W})$

Hence $S_{1}$ is the set of permutations $n 1 \ldots$ in the class which is in $1-1$ correspondence with permutations of length $n-2$ that avoid $3142,3412,3421,312,321$. These avoidance conditions amount to avoiding 312,321 alone and so this set has size $2^{n-3}$.

The equality of the enumerations in the last two lemmas appears to be no more than a coincidence. From the proofs of these lemmas it is not hard to determine the structures of the indecomposable permutations in both cases and we display these in Figure 1.

Lemma 10 For av $(2431, \mathcal{I} \mathcal{W})$ we have the enumeration formula

$$
\sum_{k=0}^{n}\left(\begin{array}{l}
n \\
k
\end{array}\right) f_{n-k}
$$

where $\left(f_{n}\right)$ is Fine's sequence A000957 in [11] (see also [6]).

Proof: $\quad$ Let $\mathcal{D}=\operatorname{av}(2431, \mathcal{I} \mathcal{W})=\operatorname{av}(2431,4231,4321)$. We shall determine the structure of a permutation $\pi \in \mathcal{D}$. Consider any left to right maximal $m$ of $\pi$, that is, any symbol larger than all of its predecessors. Since $\pi$ avoids 4231 and 4321, the subsequence of those symbols that follow $m$ in $\pi$ and are also less than $m$ avoids 231 and 321 .

Moreover, if $m^{\prime}<m$ is a right to left maximal preceding $m$ in $\pi$ then, because $\pi$ avoids 2431 , all the symbols following $m$ and less than $m^{\prime}$ must occur before any of the symbols following $m$ and greater than $m^{\prime}$ but less than $m$.

Let the sequence of left to right maximals in $\pi$ be $m_{1}, m_{2}, \ldots, m_{k}$, and let $B_{i}$ for $1 \leq i \leq k$ be the symbols of $\pi$ to the right of $m_{i}$ and between $m_{i}$ and $m_{i-1}$ in value (take $m_{0}=0$ conventionally). Since the $m$ 's are the left to right maximals, they, together with the sets $B_{i}$ partition the symbols of $\pi$. Moreover, the observation above shows that if $i<j$ then all the symbols $B_{i}$ must precede all of the symbols $B_{j}$. Figure 2 illustrates these conditions.

Every permutation of this form belongs to $\mathcal{D}$ and we can construct them all as follows. Choose an increasing sequence $m_{i}$ from among 1 through $n$. For each $i$, let $B_{i}$ be the set 


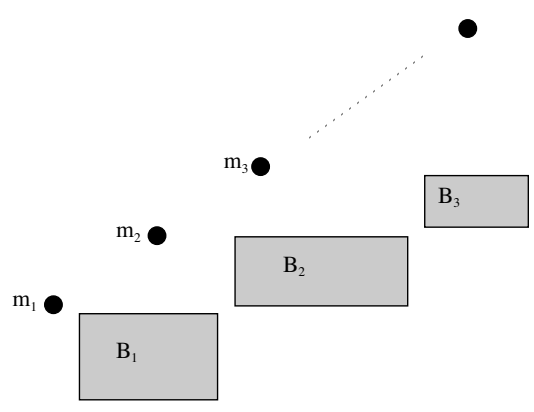

Figure 2: Structure of a permutation in $\operatorname{av}(2431, \mathcal{I} \mathcal{W})$

of values strictly between $m_{i-1}$ and $m_{i}$ and choose a $\{231,321\}$-avoiding permutation $\beta_{i}$ of $B_{i}$. Now merge the sequences $m_{1} m_{2} \cdots m_{k}$ and $\beta_{1} \beta_{2} \cdots \beta_{k}$ subject only to the condition that $m_{i}$ precedes $\beta_{i}$ for each $i$. Then the resulting permutation belongs to $\mathcal{D}$.

We say that $m_{i}$ is bound if $B_{i}$ is not empty. Otherwise, $m_{i}$ is free. A permutation in $\mathcal{D}$ is completely bound if all of its left to right maximals are bound. Consider first the completely bound permutations in $\mathcal{D}$. We associate to each of these a word in the alphabet $a, b, c$ as follows:

- Each left to right maximal is encoded by the letter $c$.

- The last symbol of each $B_{i}$ is encoded by the letter $b$.

- All remaining symbols are encoded by the letter $a$.

We note that, read left to right, the number of c's minus the number of $b$ 's is always non-negative, ends at 0 , and that an $a$ may not occur when the count is 0 . All sequences meeting these criteria can occur, and the number of permutations of $\mathcal{D}$ having all left to right maximals bound, corresponding to a sequence containing $k a$ 's is just $2^{k}$ (since each block of $a$ 's between two $b$ 's represents, together with the symbol for its final $b$, a $\{231,321\}$-avoiding permutation and there are $2^{j-1}$ such of length $j$ ). So, we can obtain a one to one correspondence between encodings and this subset of $\mathcal{D}$ if we allow the $a$ symbols to be either $a_{1}$ or $a_{2}$ arbitrarily (or by using a natural encoding of the corresponding $B$ over a two letter alphabet).

This gives a correspondence between the subset of $\mathcal{D}$ in which all left to right maximals are bound, with Motzkin paths where the horizontal steps can have either of 2 types, but may not occur on the axis, and these are enumerated by Fine's sequence $[6,11]$. Let $f_{n}$ denote its $n$th symbol.

It remains only to insert the free left to right maximals. Now observe that if we take an arbitrary $\pi \in \mathcal{D}$ and delete the free left to right maximals, what remains is indeed a completely bound permutation. Moreover, if we take such a permutation and nominate places in which left to right maximals are to be inserted freely, then there is a unique way 
to do so. That is, in a permutation belonging to $\mathcal{D}$ of length $n$ we are free to choose the number of free maximals, and their positions, and then the structure of the remaining bound permutation. This gives

$$
d_{n}=\sum_{k=0}^{n}\left(\begin{array}{l}
n \\
k
\end{array}\right) f_{n-k}
$$

as required.

Lemma 11 For av $(3241, \mathcal{I} \mathcal{W})$ we have the generating function

$$
\frac{3-13 t+2 t^{2}+5 t \sqrt{1-4 t}-\sqrt{1-4 t}}{2\left(1-4 t-t^{2}\right)}
$$

Proof: The WILFPLUS package [13] is able to produce an enumeration scheme for this class from which, in principle, one could obtain the stated generating function. However, we have derived it using techniques developed in [1].

\section{$3 \quad$ Strong sorting classes}

For weak sorting classes Proposition 3, Corollary 4 and Corollary 5 describe how the weak basis is related to the ordinary basis. The situation for strong sorting classes is considerably more complex. For example, the direct analogue of Corollary 4 is false since, for example, it would imply av $(321, \mathcal{I} \mathcal{S})=$ av $(321)$; however, $321 \mathcal{I} 3214 \mathcal{S} 3412$ and therefore $3412 \in \operatorname{av}(321) \backslash a v(321, \mathcal{I S})$. Despite this we shall prove that a strong sorting class with a finite strong basis has a finite ordinary basis and our proof will show how this ordinary basis may be computed from the strong basis.

We begin these investigations by defining three types of operation on permutations $\tau$ or their subsequences:

Switch. Exchange two symbols of $\tau$ that are currently correctly ordered.

Left. Move a symbol $t$ of $\tau$ to the left and insert some new symbol $s$ smaller than $t$ in the original position of $t$ (with appropriate renumbering of all original symbols greater than or equal to $s$ ).

Right. Move a symbol $t$ of $\tau$ to the right and insert some new symbol larger than $t$ in the original position of $t$ (also with appropriate renumbering of symbols).

It is helpful to represent a permutation $\tau$ by its graph (the set of points $(x, \tau(x)$ ) plotted in the $(x, y)$-plane) to show the effect of these operations. Our first use of this graphical representation occurs in Figure 3 which shows the effect of a single operation. We shall make heavier use of these diagrams in the proof of Theorem 14.

Suppose that $T$ is some set of permutations. Then $T$ is said to be complete if, for any $\tau \in T$, applying any of the types of operation switch, left, or right to $\tau$ results in a permutation that contains some permutation in $T$ as a subpermutation. 


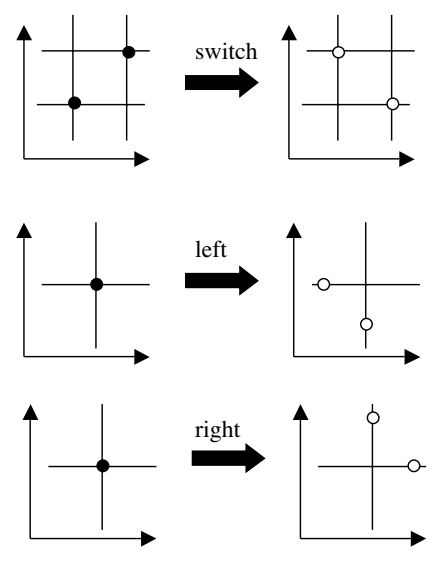

Figure 3: The operations switch, left, and right

Proposition $12 T$ is complete if and only if $\mathrm{av}(T)$ is a strong sorting class.

Proof: To begin with, assume that $T$ is complete. By definition, av $(T)$ is closed under taking subpermutations and so we must prove that it is also closed downwards in the strong order; in other words, we must prove

$$
\sigma \in \operatorname{av}(T) \text { and } \pi \mathcal{S} \sigma \Longrightarrow \pi \in \operatorname{av}(T)
$$

and it is clearly sufficient to prove this in the case that $\pi$ and $\sigma$ differ by an exchange.

So let $\sigma \in \operatorname{av}(T)$ and $\pi \mathcal{S} \sigma$ where $\pi$ and $\sigma$ differ by an exchange. For a contradiction suppose that $\pi$ contains a subsequence $p_{1} p_{2} \ldots$ order isomorphic to an element of $T$. Now $\sigma$ and $\pi$ differ only in that two symbols properly ordered in $\pi$ are in the other order within $\sigma$. If neither of these two swapped symbols are among $p_{1} p_{2} \ldots$ then $\sigma$ also contains this subsequence, and this is impossible. If both of the swapped symbols are among $p_{1} p_{2} \ldots$ then $\sigma$ contains a subsequence obtainable from $p_{1} p_{2} \ldots$ by swapping two symbols currently in the right order. But a switch operation on an element of $T$ results in a permutation that involves an element of $T$, so this is also impossible.

If only one of the swapped symbols ( $p$ say) is among $p_{1} p_{2} \ldots$ and the other symbol is, say, $q$ then consider the subsequence $\xi$ of $\sigma$ on the symbols $p_{1}, p_{2}, \ldots, q$. If, in $\pi, q$ was to the left of $p$ then we must have $q<p$. But that means that $\xi$ has been obtained from $p_{1} p_{2} \ldots$ by a left operation. Similarly if, in $\pi, q$ was to the right of $p$ then we must have $q>p$ and $\xi$ has been obtained from $p_{1} p_{2} \ldots$ by a right operation. In either case, the completeness property tells us that $\xi$ involves an element of $T$ which is impossible.

For the converse, assume that $a v(T)$ is a strong sorting class. Let $\tau$ be an arbitrary element of $T$ and suppose that $\tau^{*}$ is the result of applying a switch, left, or right operation to $\tau$. Since strong sorting classes are lower ideals in the order $\mathcal{I S}, \tau^{*} \notin \operatorname{av}(T)$. Hence $\tau^{*}$ involves an element of $T$ and therefore $T$ is complete. 
Now suppose that $X$ is a strong sorting class with strong basis $R$. Let $c(R)$ denote the ordinary basis of $X$. Our aim is to describe $c(R)$ in terms of $R$. Let $\bar{X}$ denote the complement of $X$. Then, by definition

$$
\bar{X}=\{\theta \mid \rho \mathcal{I S} \theta \text { for some } \rho \in R\} .
$$

Also, by definition, $c(R)$ is the set of minimal permutations in $\bar{X}$ (minimal with respect to $\mathcal{I}$ ). The following result shows that $c(R)$ can be constructed from $R$ by using switch, left, and right operations.

Lemma 13 Let $\theta \in c(R)$. Then there exists a sequence of permutations

$$
\theta_{0}, \theta_{1}, \ldots, \theta_{k}=\theta
$$

where $\theta_{0} \in R$, each $\theta_{i} \in c(R)$, and each $\theta_{i}$ is obtained from $\theta_{i-1}$ by a switch, left, or right operation. Furthermore, in any sequence beginning at a permutation of $R$ and ending at $\theta$ where each term arises from the previous one by a switch, left, or right operation, all permutations in the sequence are in $c(R)$.

Proof: We shall prove the first part of the lemma by induction over $\bar{X}$ with respect to the order $\mathcal{I} \mathcal{S}$. If $\theta$ happens to be minimal under $\mathcal{I} \mathcal{S}$ then, by definition, $\theta \in R$ and the result is vacuously true. This establishes the base of the induction and we now take $\theta$ to be non-minimal under $\mathcal{I S}$. In that case there exists some $\theta^{\prime} \in \bar{X}$ with $\theta^{\prime} \mathcal{I} \mathcal{S} \theta$ where this relation between $\theta^{\prime}$ and $\theta$ is a covering relation. Since $\theta$ is a minimal element for the order $\mathcal{I}$ we cannot have $\theta^{\prime} \mathcal{I} \theta$ and so we have $\theta^{\prime} \mathcal{S} \theta$; furthermore, $\theta$ can be obtained from $\theta^{\prime}$ by a switch operation (exchanging the symbols $a$ and $b$ say).

If $\theta^{\prime} \in c(R)$ then we can conclude the proof by induction; therefore assume that $\theta^{\prime} \notin c(R)$. Then there is some permutation $\theta^{\prime \prime} \in \bar{X}$ with $\theta^{\prime \prime} \mathcal{I}_{0} \theta^{\prime}$; in other words, $\theta^{\prime}$ has been obtained from $\theta^{\prime \prime}$ by inserting a new symbol $c$ (with appropriate renumbering). If $c$ is neither $a$ nor $b$ then we can interchange the switch of $a$ with $b$, and the insertion of $c$, to obtain $\theta$ by first switching $a$ and $b$ and then inserting $c$. However, that is impossible since $\theta$ is a minimal element of $\bar{X}$ under involvement.

It is now easy to see that, if $c=a$, then $\theta$ is formed from $\theta^{\prime \prime}$ by a left operation while, if $c=b$, then $\theta$ is formed from $\theta^{\prime \prime}$ by a right operation.

If $\theta^{\prime \prime} \in c(R)$ then, again, we can conclude the proof by induction. Hence, for a final contradiction, we shall assume that $\theta^{\prime \prime} \notin c(R)$. In that case there is some $\theta^{\prime \prime \prime} \in \bar{X}$ with $\theta^{\prime \prime \prime} \mathcal{I}_{0} \theta^{\prime \prime}$ and $\theta^{\prime \prime}$ is the result of inserting some new symbol $d$ into $\theta^{\prime \prime \prime}$. If $d$ is neither $a$ nor $b$ then we can obtain $\theta$ from $\theta^{\prime \prime \prime}$ by an appropriate left or right operation followed by an insertion of $d$ and, as before, this is impossible by the minimality of $\theta$.

Therefore $\{c, d\}=\{a, b\}$ (or, more precisely, the symbols that have been inserted to form $\theta^{\prime}$ from $\theta^{\prime \prime \prime}$ become $a, b$ after renumbering) and now we can obtain $\theta$ from $\theta^{\prime \prime \prime}$ by inserting $a$ and $b$ directly into their proper places within $\theta$. Again this implies that $\theta$ is not minimal and the proof of the first part is complete.

For the second part, suppose we have a sequence of permutations beginning at a permutation of $R$ and ending at $\theta$ each being generated from its predecessor by a switch, 
left, or right operation. Let $\phi$ be a permutation in this sequence that is not minimal under involvement because it has some subpermutation $\phi^{\prime} \in \bar{X}$. The switch, left, and right operations that transform $\phi$ into $\theta$ also transform $\phi^{\prime}$ and preserve the involvement property. Ultimately, this contradicts the minimality of $\theta$.

This lemma indicates how $c(R)$ can be computed from $R$ using a breadth-first search strategy. We begin from $R$ itself and apply switch, left, and right operations discarding any results that contain previously found permutations as subpermutations; and we continue using any new permutations found. We generate new permutations in order of length (by applying the operations to the smallest permutations first, and applying switch operations before left and right operations). Clearly this process will examine and not discard every $I$-minimal permutation of $\bar{X}$. On the other hand, any permutation $\sigma$ which is not $I$ minimal will contain a (shorter) $I$-minimal permutation $\tau$ which, by Lemma 13 and the choice of search strategy, will have been examined before $\sigma$ (and not discarded). By virtue of the presence of $\tau, \sigma$ will be discarded. Once no new permutations can be generated we will have found a complete set and, by Lemma 13, this will be $c(R)$. Our next result shows that this process terminates if $R$ is finite.

Theorem 14 Let $X$ be a strong sorting class with strong basis $R$ and suppose that $R$ is finite. Then $c(R)$ is also finite.

Proof: We shall be relying on Lemma 13 which proves that every permutation $\theta \in c(R)$ can be constructed from some permutation in $R$ by a sequence of switch, left, and right operations. In the first part of the proof we shall show that $\theta$ can be constructed by a sequence in which all the left operations precede all the right operations and, in turn, all the right operations precede all the switch operations. The graphical representations of switch, left, and right introduced previously will be used extensively.

Suppose in the sequence of operations that has realised $\theta$ we have a switch operation followed by a left operation. If the left operation was applied to neither of the two symbols that took part in the switch operation then it is evident that the same effect can be achieved by a left operation followed by a switch. However, if the left operation was applied to one of the two switched symbols, we must argue more carefully. Diagrammatically we have one of four different cases as shown in Figure 4. Each of these cases can be modified as shown in Figure 5 so that the same effect is obtained by a left operation followed by a switch operation.

A similar argument shows that any switch operation followed by a right operation may also be replaced by a right operation followed by a switch. Therefore the sequence of operations may be assumed to have all the switch operations at the end.

Now suppose that in the realisation of $\theta$ there is a right operation followed by a left operation. If the two symbols generated by the right operation are not affected by the left operation then these two operations can obviously be interchanged. In the contrary case there are, again, four cases as shown in Figure 6. The second and fourth of these are impossible because their result is not a minimal permutation: they each involve a permutation arising from a different right operation on the initial configuration. The first 


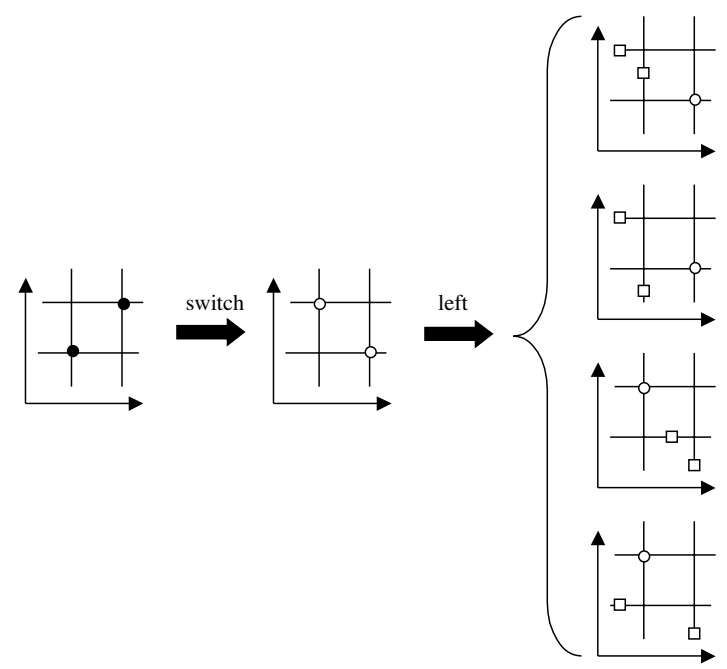

Figure 4: A switch operation followed by a left operation

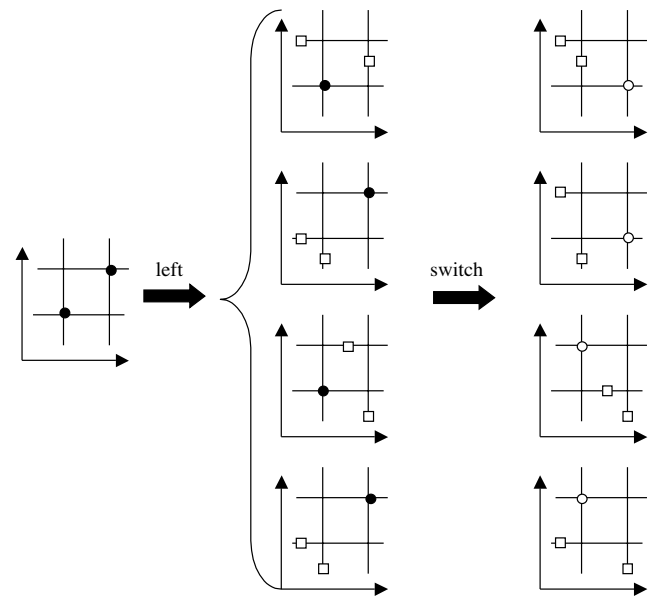

Figure 5: The same effect with left followed by switch 


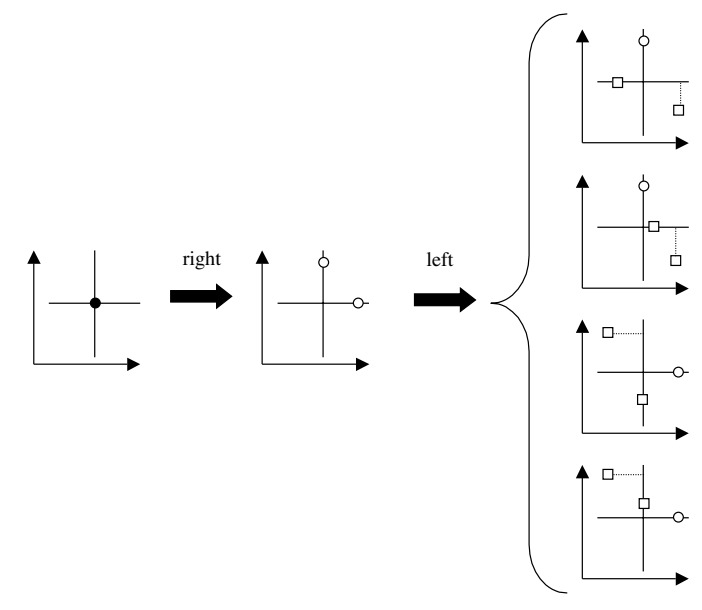

Figure 6: right followed by left: different cases (two impossible)

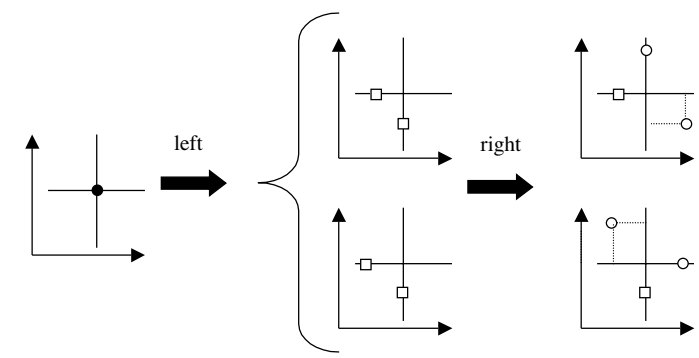

Figure 7: The same effect with left followed by right

and third can be achieved by a left operation then a right operation; the intermediate configurations are shown in Figure 7.

Now suppose that $\theta \in c(R)$. We take a sequence of left, right and switch operations that generate $\theta$ from some $\rho \in R$. By the results above we may assume that all the left operations are applied first, followed by all the right operations, and finally the switch operations. We can regard each left operation as one which splits a point of the diagram into two, moves one of them to the left and the other one down. If we have two left operations, the second of which splits one of the points created by the first left (as in Figure 8) the result is not minimal since it involves a permutation formed by performing one left operation only. On the other hand two left operations that are not so linked can be commuted. Thus, no new point gets split by another left operation and so the number of left operations cannot be more than the original number of points present. Hence the series of left operations cannot increase the length by more than a factor of 2 . The same is true of the right operations and so $|\theta| \leq 4|\rho|$ which completes the proof. 


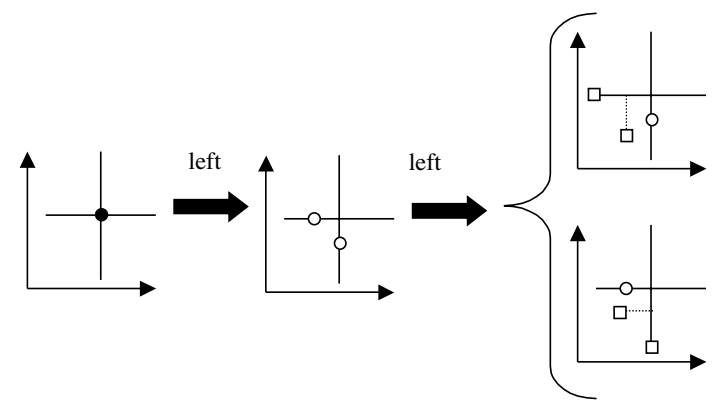

Figure 8: Two left operations

We turn now to the enumeration problem for strong sorting classes whose strong basis is finite and give some sample results. Our general method is to first determine the ordinary basis of the class by the process described above and use our experience in closed class enumeration.

As an example of a fairly typical situation we note that

$$
\begin{aligned}
c(\{4231\})= & \{4231,4321,35142,45312,42513,45132,35412, \\
& 45213,43512,456123,351624,451623,356124\} .
\end{aligned}
$$

The next two results summarise the enumerations of all strong sorting classes with a single strong basis permutation of length 3 or 4 (omitting trivial cases or cases that follow from symmetry).

Proposition 15 For a single strong basis permutation of length 3

Basis permutation Ordinary basis Enumeration

\begin{tabular}{lll}
\hline \hline 312 & $\{321,312\}$ & $2^{n-1}$ \\
\hline 321 & $\{321,3412\}$ & $a_{n}=3 a_{n-1}-a_{n-2}$ for $n \geq 3$
\end{tabular}

Proof: The ordinary basis can be confirmed using Proposition 12 and the enumerations are well-known.

Proposition 16 For a single strong basis permutation of length 4 


\begin{tabular}{lll} 
Name & Basis permutation & Enumeration \\
\hline \hline$I$ & 1234 & 0 for $n \geq 4$ \\
\hline$I I$ & 1243 & 6 for $n \geq 4$ \\
\hline$I I I$ & 1324 & 4 for $n \geq 4$ \\
\hline$I V$ & 1342 & $3 \times 2^{n-2}$ for $n \geq 3$ \\
\hline$V$ & 1432 & $a_{n}=3 a_{n-1}-a_{n-2}$ for $n \geq 4$ \\
\hline$V I$ & 2143 & $a_{n}=4 n-6$ for $n \geq 2$ \\
\hline$V I I$ & 2341 & $2 \times 3^{n-2}$ for $n \geq 2$ \\
\hline$V I I I$ & 2413 & $a_{n}=3 a_{n-1}-2 a_{n-2}+2 a_{n-3}$ for $n \geq 4$ \\
\hline$I X$ & 2431 & $a_{n}=4 a_{n-1}-3 a_{n-2}+2 a_{n-3}$ for $n \geq 4$ \\
\hline$X$ & 3412 & $a_{n}=4 a_{n-1}-2 a_{n-2}$ for $n \geq 3$ \\
\hline$X I$ & 3421 & $\left(4^{n}+2\right) / 3$ for $n \geq 2$ \\
\hline$X I I$ & 4231 & $a_{n}=4 a_{n-1}-2 a_{n-2}+4 a_{n-3}-a_{n-5}$ for $n \geq 6$ \\
\hline$X I I I$ & 4321 & $a_{n}=4 a_{n-1}+a_{n-2}+a_{n-3}-4$ for $n \geq 5$
\end{tabular}

Proof: We give a sketch of the proof of the last of these only. The form of the other proofs is similar although the details vary considerably. First of all we use the completion process to determine the basis of av $(4321, \mathcal{I S})$. This turns out to be

$$
\{4321,45132,45231,35412,53412,45213,43512,45312,456123,451623,356124\} \text {. }
$$

Next we observe that the basis elements 4321,45132,45231,45213,45312, 456123 show every permutation of the class has a 1 or 2 or 3 in the first 3 places. Denote the number of permutations of length $n$ in the class by $a_{n}$. In the following case analysis we use the letter $c$ to stand for any symbol larger than 2, and the letter $d$ for any symbol larger than 3 .

The situation for the permutations that have 1 or 2 in their first or second positions is summarised by

\begin{tabular}{lll} 
Type & Enumeration & Explanation \\
\hline $1 \alpha$ & $a_{n-1}$ & $\alpha$ can be arbitrary \\
$2 \alpha$ & $a_{n-1}$ & $\alpha$ can be arbitrary \\
$c 1 \alpha$ & $a_{n-1}-a_{n-2}$ & $c \alpha$ is arbitrary but cannot start with 2 \\
$c 2 \alpha$ & $a_{n-1}-a_{n-2}$ & $c \alpha$ is arbitrary but cannot start with 1
\end{tabular}

From now on we assume the first two places do not contain a 1 or a 2 . The next cases are those where 3 also does not occur in the first two positions but one of 1,2,3 is in the third position. Their forms are as follows

\begin{tabular}{lll} 
Type & Enumeration & Explanation \\
\hline$d d 1 \alpha$ & $2 a_{n-3}-2$ & Discussed below \\
$d d 2 \alpha$ & 0 & Uses 4321, 45213 and 45231 \\
$d d 3 \alpha$ & 0 & Uses 4321 and 45312
\end{tabular}

With symbol 3 in second place we have the cases: 


\begin{tabular}{lll} 
Type & Enumeration & Explanation \\
\hline$d 31 \alpha$ & $a_{n-2}-a_{n-3}$ & By removing 1, in correspondence with the type $c 2 \alpha$ \\
$d 32 \alpha$ & 0 & Uses 4321 \\
$d 3 d \alpha$ & 0 & Uses 4321,43512 and 53412
\end{tabular}

With symbol 3 in first place we have the cases:

\begin{tabular}{lll} 
Type & Enumeration & Explanation \\
\hline $3 d 1 \alpha$ & $a_{n-2}-a_{n-3}$ & By removing 1, in correspondence with the type $c 2 \alpha$ \\
$3 d 2 \alpha$ & $a_{n-2}-a_{n-3}$ & By removing 2, in correspondence with the type $c 2 \alpha$ \\
$3 d d \alpha$ & $2 a_{n-3}-2$ & Discussed below
\end{tabular}

The explanations above are straightforward except for the two where we promised further discussion. For the first of these (the type $d d 1 \alpha$ ) we can prove (by a quite lengthy case by case examination whose details we omit) that $\alpha$ starts with 2 . Let $b_{n}$ be the number of permutations of this type. By removing the symbol 2 we obtain a correspondence with the type $c c 1 \alpha$ of length $n-1$. The latter sequences have one of the forms $3 d 1 \alpha$ ( $a_{n-3}-a_{n-4}$ of them), $d 31 \alpha$ (also $a_{n-3}-a_{n-4}$ of them), or $d d 1 \alpha$ ( $b_{n-1}$ of them). Hence $b_{n}=b_{n-1}+2\left(a_{n-3}-a_{n-4}\right)$. Iterating this recurrence leads to $b_{n}=b_{5}+2 a_{n-3}-2 a_{2}$ and since $b_{5}=a_{2}=2$ the required result follows. The second case where further discussion was promised is the $3 d d \alpha$ case. Here we can prove that the sequences are of the form $34 d \alpha$ and then we argue in a similar way.

Adding together all these contributions we obtain $a_{n}=4 a_{n-1}+a_{n-2}+a_{n-3}-4$.

The tenor of the above results hints that the theory of strong sorting classes is going to be more complex than that for weak sorting classes. However, in the remainder of this section we give some compensatory results which go some way to proving that it may actually be less complex.

Consider the following family of closed classes. The closed class $\mathcal{B}(r, s)$ is defined by the $r ! s !$ (ordinary) basis permutations $\beta \alpha$ where $|\beta|=r,|\alpha|=s$ and every symbol of $\beta$ is greater than every symbol of $\alpha$. It follows directly from the definition that, for a permutation $\pi$ of length $n$ to be a member of $\mathcal{B}(r, s)$, there must not exist subsets $I, J \subseteq\{1, \ldots, n\}$ such that $|I| \geq r,|J| \geq s, I<J$ and $\pi(I)>\pi(J)$. Two other readily checked properties are

$$
\mathcal{B}(r, s)^{*}=\mathcal{B}(r, s)^{-1}=\mathcal{B}(s, r) .
$$

As a first application of Proposition 12 we have

Lemma $17 \mathcal{B}(r, s)$ is a strong sorting class. Indeed, if

$$
\theta_{r s}=s+1, s+2, \ldots, s+r, 1,2, \ldots, s
$$

then

$$
\mathcal{B}(r, s)=\operatorname{av}\left(\theta_{r s}, \mathcal{I} \mathcal{S}\right)
$$

Proof: It is readily checked that the basis of $\mathcal{B}(r, s)$ is complete so the first part follows from Proposition 12. For the second part we note that, as $\mathcal{B}(r, s)$ is a strong sorting class 
not containing $\theta_{r s}$ (which is one of its basis permutations), $\mathcal{B}(r, s) \subseteq$ av $\left(\theta_{r s}, \mathcal{I} \mathcal{S}\right)$. On the other hand every basis permutation $\beta$ of $\mathcal{B}(r, s)$ satisfies $\theta_{r s} \mathcal{S} \beta$ and hence cannot be involved in any permutation of $\operatorname{av}\left(\theta_{r s}, \mathcal{I} \mathcal{S}\right)$; so $\operatorname{av}\left(\theta_{r s}, \mathcal{I} \mathcal{S}\right) \subseteq \mathcal{B}(r, s)$.

The importance of the strong sorting classes $\mathcal{B}(r, s)$ stems from

Proposition 18 Every proper strong sorting class is contained in some $\mathcal{B}(r, r)$.

Proof: Let $X$ be a strong sorting class contained in no $\mathcal{B}(r, r)$. Then $X$ contains permutations of the form $\beta \alpha$ where $\beta>\alpha$ and $|\alpha|=|\beta|=r$ for all values of $r$. But, if $X$ contains say $b_{1} \ldots b_{r} a_{1} \ldots a_{r}$ with all $b_{i}>a_{j}$, then by a series of exchanges of the form $b_{i} \leftrightarrow a_{j}$ we can produce a permutation with any rearrangement of $a_{1} \ldots a_{r}$ in the first $r$ positions. Thus $X$ contains every permutation of length $r$ and so contains every permutation.

This proposition indicates that the classes $\mathcal{B}(r, s)$ are going to be fundamental in the understanding of strong sorting classes. Obviously, $\mathcal{B}(1,1)$ consists only of identity permutations. The first non-trivial cases are $\mathcal{B}(1,2)$ and $\mathcal{B}(2,1)$ whose structure is given next. Subsequently, in Theorem 23, we shall give a complete description of the classes $\mathcal{B}(r, s)$.

Lemma $19 \mathcal{B}(1,2)$ consists of permutations whose cycle structure is

$$
\left(1,2, \ldots, k_{1}\right)\left(k_{1}+1, k_{1}+2, \ldots, k_{2}\right)\left(k_{2}+1 \ldots\right) \ldots
$$

and $\mathcal{B}(2,1)$ is the class of their inverses.

Proof: $\mathcal{B}(1,2)$ is the class of $\{321,312\}$-avoiding permutations whose structure is wellknown. The second statement follows from $\mathcal{B}(2,1)=\mathcal{B}(1,2)^{-1}$.

The classes $\mathcal{B}(r, s)$ were also defined (somewhat differently) by Mansour and Vainshtein [10] who enumerated them by generating functions. The following result gives an elementary method of enumerating them.

Proposition 20 Let $x_{n}$ be the number of permutations of length $n$ in $\mathcal{B}(r, s)$. Then

$$
x_{n}=r s x_{n-1}-2 !\left(\begin{array}{l}
r \\
2
\end{array}\right)\left(\begin{array}{l}
s \\
2
\end{array}\right) x_{n-2}+3 !\left(\begin{array}{l}
r \\
3
\end{array}\right)\left(\begin{array}{l}
s \\
3
\end{array}\right) x_{n-3}-\ldots
$$

Proof: Suppose we have a permutation of $\{1,2, \ldots, n\} \backslash\{t\}$ where $t$ is one of $\{n, n-$ $1, \ldots, n-r+1\}$ and that the permutation is in (i.e. order isomorphic to a permutation of) $\mathcal{B}(r, s)$. If we insert the symbol $t$ anywhere within the final $s$ symbols of this permutation we cannot introduce a subpermutation isomorphic to a basis permutation of $\mathcal{B}(r, s)$, so the result is still in $\mathcal{B}(r, s)$.

Now consider the possible forms of a permutation of length $n$ in $\mathcal{B}(r, s)$. Such a permutation must have at least one of the $r$ largest symbols somewhere within its last $s$ positions. The choice of the value of this symbol together with its positions, and the results 
of the previous paragraph, would appear to give $r s x_{n-1}$ permutations in $\mathcal{B}(r, s)$ of length $n$. However, this overcounts the permutations which have two or more of their $r$ largest symbols in their final $s$ positions. So we seem to have $r s x_{n}-2$ ! $\left(\begin{array}{c}r \\ 2\end{array}\right)\left(\begin{array}{l}s \\ 2\end{array}\right) x_{n-2}$ permutations. However, this undercounts by $3 !\left(\begin{array}{l}r \\ 3\end{array}\right)\left(\begin{array}{l}s \\ 3\end{array}\right) x_{n-3}$ the permutations with three or more of their $r$ largest symbols in their final $s$ positions. Continuing by inclusion-exclusion we obtain the formula.

In $[2]$ it was observed that, for closed classes $\mathcal{X}, \mathcal{Y}$, the set of permutation products

$$
\mathcal{X} \circ \mathcal{Y}=\{\alpha \circ \beta \mid \alpha \in \mathcal{X}, \beta \in \mathcal{Y}\}
$$

was also a closed class. A similar result holds for strong sorting classes.

Proposition 21 If $\mathcal{X}$ and $\mathcal{Y}$ are strong sorting classes so also is $\mathcal{X} \circ \mathcal{Y}$.

Proof: A class $\mathcal{Z}$ is closed under the strong order if and only if for all $\zeta \in \mathcal{Z}$ and all $i, j$ with $i<j$ and $i^{\zeta}>j^{\zeta}$ we have (using cycle notation for transpositions)

$$
(i, j) \circ \zeta \in \mathcal{Z}
$$

Now let $\alpha \in \mathcal{X}$ and $\beta \in \mathcal{Y}$ and put $\gamma=\alpha \circ \beta$. Suppose $i<j$ and $i^{\gamma}>j^{\gamma}$. Then $(i, j) \circ \gamma=(i, j) \circ \alpha \circ \beta$. Either $i^{\alpha}>j^{\alpha}$ in which case $(i, j) \circ \alpha \in \mathcal{X}$ or $i^{\alpha}<j^{\alpha}$ in which case

$$
(i, j) \circ \alpha \circ \beta=\alpha \circ \alpha^{-1} \circ(i, j) \circ \alpha \circ \beta=\alpha \circ\left(i^{\alpha}, j^{\alpha}\right) \circ \beta
$$

and $\left(i^{\alpha}, j^{\alpha}\right) \circ \beta \in \mathcal{Y}$ since $i^{\alpha \beta}>j^{\alpha \beta}$.

Lemma $22 \mathcal{B}(1, q) \supseteq \mathcal{B}(1,2)^{q-1}$ and $\mathcal{B}(p, 1) \supseteq \mathcal{B}(2,1)^{p-1}$.

Proof: If the first part were false there would be some basis element of $\mathcal{B}(1, q)$ which was expressible as a product of $q-1$ elements of $\mathcal{B}(1,2)$. Such a basis element has length $q+1$ and maps 1 to $q+1$ (i.e. as a sequence it begins with $q+1$ ). However each element of $\mathcal{B}(1,2)$ maps symbols $t$ either to $t+1$ or to a smaller symbol (Lemma 19) and so a product of $q-1$ of them cannot map 1 to $q+1$. The second part follows by taking inverses.

\section{Theorem 23}

$$
\mathcal{B}(p, q)=\mathcal{B}(2,1)^{p-1} \circ \mathcal{B}(1,2)^{q-1}
$$

and

$$
\mathcal{B}(p, q) \circ \mathcal{B}(r, s)=\mathcal{B}(p+r-1, q+s-1)
$$

Proof: We prove a series of results from which we then deduce the theorem.

$\mathrm{A}:(X \circ Y)^{*}=X^{*} \circ Y^{*}$. 
If $\alpha, \beta$ have length $n$ and $\rho$ is the reverse identity permutation then

$$
\begin{aligned}
(\alpha \circ \beta)^{*} & =\rho \circ \alpha \circ \beta \circ \rho \\
& =\rho \circ \alpha \circ \rho \circ \rho \circ \beta \circ \rho \\
& =\alpha^{*} \circ \beta^{*} .
\end{aligned}
$$

B: $\mathcal{B}(p, q) \supseteq \mathcal{B}(p, 1) \circ \mathcal{B}(1, q)$.

If this is false there would exist a permutation $\pi=\alpha \beta$ with $\alpha \in \mathcal{B}(p, 1), \beta \in \mathcal{B}(1, q)$ and sets $I, J$ with $|I| \geq p,|J| \geq q, I<J$ and $\pi(I)>\pi(J)$. Then, as $\beta(\alpha(I))>\beta(\alpha(J))$ we have

$$
\beta(r)>\beta(s)
$$

for all $r \in \alpha(I), s \in \alpha(J)$. For each fixed $r \in \alpha(I), r<\alpha(J)$ is impossible since $\beta \in \mathcal{B}(1, q)$. Thus, for all $r \in \alpha(I)$, we have $r>s_{0}$ where $s_{0}$ is the minimal value in $\alpha(J)$. But now, writing $j=\alpha^{-1}\left(s_{0}\right)$ we have $I<j$ and $\alpha(I)>\alpha(j)$ contradicting that $\alpha \in \mathcal{B}(p, 1)$.

C: $\mathcal{B}(p, q) \subseteq \mathcal{B}(2,1) \circ \mathcal{B}(p-1, q)$.

We say that a subsequence of length $r+s$ is of type $(r, s)$ if its initial $r$ symbols are all greater than the final $s$ symbols. Let $\sigma \in \mathcal{B}(p, q)$. By definition $\sigma$ has no subsequences of type $(p, q)$. A subsequence $\gamma$ of $\sigma$ is defined to be critical if

1. $|\gamma|=p-1$, and

2. the set $S(\gamma)$ of symbols that follow $\gamma$ and are less than every symbol of $\gamma$ has size at least $q$.

The critical subsequences $\gamma$ together with the $q$-symbol subsequences of $S(\gamma)$ comprise all the subsequences of $\sigma$ of type $(p-1, q)$.

Notice that two distinct critical sequences $\gamma_{1}, \gamma_{2}$ cannot have the same last symbol. For then $\gamma_{1} \cup \gamma_{2}$ would have size at least $p$ and the minimal symbol of this subsequence would be one of $\min \left(\gamma_{1}\right)$ and $\min \left(\gamma_{2}\right)$ and so this subsequence, together with either $S\left(\gamma_{1}\right)$ or $S\left(\gamma_{2}\right)$ would contain a subsequence of type $(p, q)$. We can therefore order the critical sequences by the position in $\sigma$ of their final symbols.

Let $\gamma$ be any critical sequence with final symbol $g$ and let $x_{r}, x_{r-1}, \ldots, x_{q}, \ldots, x_{1}$ be the symbols of $S(\gamma)$ in order of their occurrence in $\sigma$. There can be no symbols of $\sigma$ between $g$ and $x_{q}$ except for $x_{r}, \ldots, x_{q+1}$. For if there were such a symbol it could not be smaller than $\min (\gamma)$ (or, by definition, it would be in $S(\gamma)$ ) and, if it were larger than $\min (\gamma)$ then together with $\gamma$ and $x_{q}, \ldots, x_{1}$ we would have a subsequence of type $(p, q)$. Notice now that if the segment $g x_{r} x_{r-1} \cdots x_{q}$ was replaced by $x_{r} x_{r-1} \cdots x_{q} g$ then we would destroy all the subsequences of type $(p-1, q)$ that stemmed from $\gamma$; moreover no further subsequences of type $(p-1, q)$ would be introduced. This replacement can be effected by pre-multiplication of $\sigma$ by a cycle $(i, i+1, \ldots, j)$ where $i$ and $j$ are the initial and final positions of the segment $g x_{r} x_{r-1} \cdots x_{q}$.

Now, the next critical sequence $\gamma^{\prime}$ (which ends after $g$ ) cannot have final symbol one of $x_{r}, x_{r-1}, \ldots, x_{q}$. If it did then $\gamma \cup \gamma^{\prime}$ together with $S\left(\gamma^{\prime}\right)$ would contain a subsequence of 
type $(p, q)$. Therefore the next critical sequence has a final symbol which occurs after $x_{q}$. That shows that the subsequences of type $(p-1, q)$ that stem from $\gamma^{\prime}$ can be destroyed by pre-multiplication by a similar cycle disjoint from the one defined above.

Hence all the subsequences of type $(p-1, q)$ can be destroyed by pre-multiplication by a product of disjoint cycles of the form given above. But such a product is, by Lemma 19, a permutation $\alpha \in \mathcal{B}(1,2)$. So there exists some $\alpha \in \mathcal{B}(1,2)$ for which $\alpha \sigma \in \mathcal{B}(p-1, q)$ and, as $\mathcal{B}(1,2)^{-1}=\mathcal{B}(1,2)$ we have $\sigma \in \mathcal{B}(2,1) \circ \mathcal{B}(p-1, q)$ as required.

$\mathrm{D}: \mathcal{B}(p, q)=\mathcal{B}(p, 1) \circ \mathcal{B}(1, q)=\mathcal{B}(2,1)^{p-1} \circ \mathcal{B}(1,2)^{q-1}$.

By repeated application of $C$ we have

$$
\mathcal{B}(p, q) \subseteq \mathcal{B}(2,1)^{p-1} \circ \mathcal{B}(1, q) \subseteq \mathcal{B}(p, 1) \circ \mathcal{B}(1, q)
$$

(the second inclusion following from Lemma 22) and the result follows from B.

$\mathrm{E}: \mathcal{B}(p, 1) \circ \mathcal{B}(r, 1)=\mathcal{B}(p+r-1,1)$ and $\mathcal{B}(1, q) \circ \mathcal{B}(1, s)=\mathcal{B}(1, q+s-1)$.

This follows immediately from $\mathrm{D}$ since $\mathcal{B}(1,1)$ is the class of identity permutations.

$\mathrm{F}: \mathcal{B}(p, 1) \circ \mathcal{B}(1, q)=\mathcal{B}(1, q) \circ \mathcal{B}(p, 1)$.

The left hand side is $\mathcal{B}(p, q)$ (by $\mathrm{D}$ ) and the right hand side is

$$
\begin{aligned}
\mathcal{B}(1, q) \circ \mathcal{B}(p, 1) & =\mathcal{B}(q, 1)^{*} \circ \mathcal{B}(1, p)^{*} \\
& =(\mathcal{B}(q, 1) \circ \mathcal{B}(1, p))^{*} \\
& =\mathcal{B}(q, p)^{*} \\
& =\mathcal{B}(p, q) .
\end{aligned}
$$

We can now complete the proof since

$$
\begin{aligned}
\mathcal{B}(p, q) \circ \mathcal{B}(r, s) & =\mathcal{B}(p, 1) \circ \mathcal{B}(1, q) \circ \mathcal{B}(r, 1) \circ \mathcal{B}(1, s) \\
& =\mathcal{B}(p, 1) \circ \mathcal{B}(r, 1) \circ \mathcal{B}(1, q) \circ \mathcal{B}(1, s) \\
& =\mathcal{B}(p+r-1,1) \circ \mathcal{B}(1, q+s-1) \\
& =\mathcal{B}(p+r-1, q+s-1) .
\end{aligned}
$$

Finally we mention another respect in which strong sorting classes are more tractable than weak sorting classes. It is no accident that the enumerations in Propositions 15 and 16 were all rational since in [1] techniques are given to show that every finitely based subclass of $\mathcal{B}(r, s)$ has a rational generating function. Consequently, using Theorem 14 we have

Theorem 24 Every strong sorting class whose strong basis is finite has a rational generating function.

Acknowledgement We made extensive use of the GAP system [5] in deriving some of the enumerative results. We also thank the referee whose very careful reading of the paper resulted in many improvements. 


\section{References}

[1] M. H. Albert, S. A. Linton, N. Ruškuc: The insertion encoding of permutations, Department of Computer Science, University of Otago, Technical Report OUCS-2005-05 (see http://www.cs.otago.ac.nz/research/publications/oucs-2005-05.pdf).

[2] M. H. Albert, R. E. L. Aldred, M. D. Atkinson, H. van Ditmarsch, C. C. Handley, D. A. Holton, D. J. McCaughan: Compositions of pattern restricted sets of permutations, in preparation.

[3] M. D. Atkinson, D. Tulley, M. J. Livesey: Permutations generated by token passing in graphs, Theoretical Computer Science 178 (1997), 103-118.

[4] M. D. Atkinson, T. Stitt: Restricted permutations and the wreath product, Discrete Math. 259 (2002), 19-36.

[5] The GAP Group, GAP - Groups, Algorithms, and Programming, Version 4.4; 2004, (http://www.gap-system.org).

[6] E. Deutsch, L. Shapiro: A survey of the Fine numbers, Discrete Math. 241 (2001), $1-3$.

[7] I. M. Gessel: Symmetric functions and P-recursiveness, J. Combin. Theory Ser. A 53 (1990), 257-285.

[8] D. E. Knuth: Fundamental Algorithms, The Art of Computer Programming Vol. 1 (Second Edition), Addison-Wesley, Reading, Mass. (1973).

[9] D. Kremer: Permutations with forbidden subsequences and a generalised Schröder number, Discrete Math. 218 (2000),121-130.

[10] T. Mansour, A. Vainshtein: Avoiding maximal parabolic subgroups of $S_{k}$, Discrete Mathematics and Theoretical Computer Science 4 (2000), 67-77.

[11] N. J. A. Sloane: Superseeker, superseeker@research.att.com.

[12] Special issue on permutation patterns, Electron. J. Combin. 9(2) (2003), editors M. D. Atkinson, D. A. Holton.

[13] V. Vatter: WILFPLUS, Maple package available at http://www.math.rutgers . edu/ vatter/programs/wilfplus/index.html.

[14] D. Zeilberger: Enumeration Schemes, and More Importantly, Their Automatic Generation, Annals of Combinatorics 2 (1998), 185-195. 